\title{
psiTurk: An open-source framework for conducting replicable behavioral experiments online
}

\author{
Todd M. Gureckis ${ }^{1}$ - Jay Martin ${ }^{1,2}$ • John McDonnell ${ }^{1,3}$ • Alexander S. Rich ${ }^{1}$. \\ Doug Markant ${ }^{4}$ - Anna Coenen ${ }^{1}$. David Halpern ${ }^{1}$ - Jessica B. Hamrick ${ }^{5}$. \\ Patricia Chan ${ }^{1}$
}

Published online: 1 October 2015

(C) Psychonomic Society, Inc. 2015

\begin{abstract}
Online data collection has begun to revolutionize the behavioral sciences. However, conducting carefully controlled behavioral experiments online introduces a number of new of technical and scientific challenges. The project described in this paper, psiTurk, is an open-source platform which helps researchers develop experiment designs which can be conducted over the Internet. The tool primarily interfaces with Amazon's Mechanical Turk, a popular crowd-sourcing labor market. This paper describes the basic architecture of the system and introduces new users to the overall goals. psiTurk aims to reduce the technical hurdles for researchers developing online experiments while improving the transparency and collaborative nature of the behavioral sciences.
\end{abstract}

psiTurk was primarily written by the listed authors at the time the first draft of this paper was constructed (version 2.0.0). However, many people continually contribute to psiTurk's evolving code base and documentation via GitHub. Illustrations by Kylan Larson (http://kylanlarson.com).

Todd M. Gureckis

todd.gureckis@nyu.edu

1 Department of Psychology, New York University, New York, NY, USA

2 Stitch Fix, Inc., San Francisco, CA, USA

3 Square, Inc., San Francisco, CA, USA

4 Center for Adaptive Rationality, Max Planck Institute for Human Development, Berlin, Germany

5 Department of Psychology, University of California at Berkeley, Berkeley, CA, USA
Keywords Online experiments · Open science · Amazon Mechanical Turk $\cdot$ Internet experiments $\cdot$ psiTurk

\section{Introduction}

The ability to collect behavioral data from a large group of individuals anonymously over the Internet has transformed the behavioral and social sciences. In addition to large scale experiments such as those conducted by websites such as Facebook or Twitter (e.g., Chen \& Sakamoto, 2013; Kramer et al., 2014 ; Wu et al., 2011), many researchers use online data collection to deploy "traditional" experimental psychology tasks. The benefit of the online versions of traditional behavioral experiments include faster data collection and access to a potentially more diverse pool of participants. At the same time, conducting experiments online introduces a number of new technical and scientific challenges that must be addressed (Reips, 2000; Birnbaum, 2004; Reips, 2002).

The project described in this paper, psiTurk, is an opensource project that aims to address many of the technical challenges involved in deploying psychology experiments online. It facilitates the creation, deployment, sharing, and replication of web-based experiments. Study participants can be recruited via Amazon Mechanical Turk (AMT), which is currently the most popular platform for running paid human experiments online.

To briefly summarize, psiTurk provides a Python-based web server and associated JavaScript library for conducting experiments on the web, saving experimental data to a database, and restricting the participant pool according to the experimenter's needs (e.g., to prevent from repeating a study). It also provides a command-line interface with AMT to create and test new projects as well as to manage and pay 


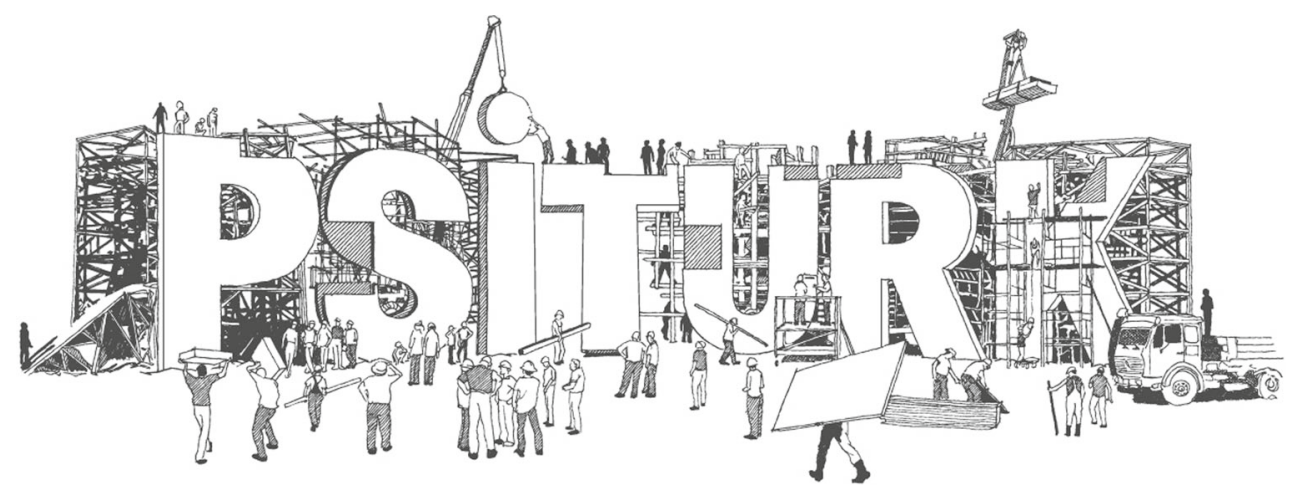

Fig. 1 The psiTurk project is hosted on GitHub (https://github.com/ NYUCCL/psiTurk) and at its main webpage (https://psiturk.org). The commissioned logo reflects the community-built orientation of the

participants. Finally, in the spirit of the recent "open science" movement (Open Science Collaboration, 2012), it provides an open-source Experiment Exchange for sharing experiments that can be easily replicated and extended by other researchers. The code for psiTurk is open source, hosted publicly at https://github.com/NYUCCL/psiTurk, and interested researchers can easily contribute to improvements and bug fixes (see Fig. 1).

The principal goal of psiTurk is to handle common technical challenges so that researchers can focus on the development and replication of online experiments. In this paper, we present a high-level overview of the psiTurk framework and its advantages for running web-based experiments. In supplement to this overview, psiTurk provides a large and continually evolving set of documentation hosted online (https://psiturk.org/docs). We begin with an overview of the issues involved in online behavioral research, the AMT platform, and the specific challenges that psiTurk is designed to address. We then step through the core functionality of psiTurk and describe its role from the point of view of both the experimenter and the participant. Finally, we consider some future directions for psiTurk.

Note that an exhaustive discussion of all the caveats, challenges, and opportunities presented by web-based experimentation lies beyond the scope of this paper (see e.g., Reips, 2000; Birnbaum, 2004; Reips, 2002). Rather, we will focus on the specific methodological contributions of psiTurk and its possibilities in the future.

\section{The challenges of web-based experimental behavioral research}

It is easy to see why online experiments are an attractive option for behavioral scientists (see also, Birnbaum 2004). Compared to traditional methods of experimentation in the laboratory, they allow researchers to collect large data sets in a fraction of the time and at much lower cost (in terms of project. A core philosophy is that better scientific software can be developed with more diverse input from many programmers (Raymond, 1999)

time, employment of research assistants, and typical compensation costs for participants). Designing experiments for the web allows researchers to leverage a large set of software libraries, such as Node.js, jQuery, d3.js, and Bootstrap. These libraries were developed to assist in the development of commercial web applications and can be used to design the look and flow of an experiment in ways that far exceed the capabilities of traditional experiment software developed in academia. Additionally, such tools enable a much wider range of experiments, including more complicated interactive interfaces, or even multi-player games.

Still, there are a number of technical challenges to running experiments online (for a more in-depth discussion of these challenges, see Reips, 2002). For example, unlike in a laboratory setting, researchers have to deal with setting up a web server, recording data in a robust and secure way, and compensating participants over the Internet. Prior to the release of psiTurk version 2.0.0 (early 2014), we conducted an informal survey of behavioral researchers about their attitudes, concerns, and needs regarding online experimentation. To help introduce some of the core features and goals of psiTurk we quickly summarize some of the findings from this survey.

\section{Informal survey of the challenges researchers face conducting online experiments}

We collected responses from 201 researchers in psychology (58\%), linguistics (16\%), marketing (7\%), neuroscience $(6 \%)$, and economics (4\%), most of whom had some experience collecting behavioral data online in their labs $(85 \%)$. Respondents were recruited via mailing lists devoted to behavioral science and psychology as well as blogs and social media.

The survey questions asked respondents about their attitudes and opinions towards online data collection as well as the features they would look for in a software package that helps with online data collection. In the interest of 


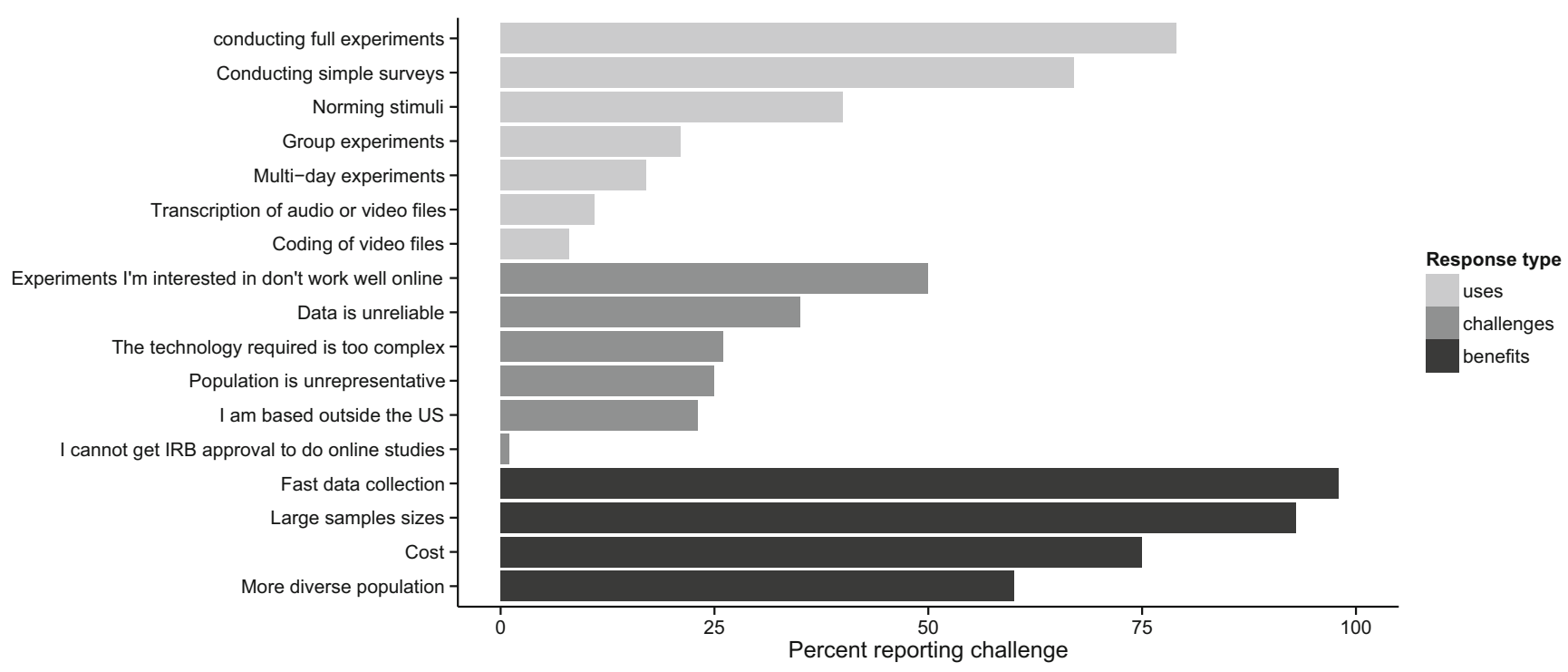

Fig. 2 Reported uses, challenges, and benefits of online data collection in a survey of 201 behavioral researchers

brevity, we highlight some of the most important questions and answers. ${ }^{1}$

Survey respondents showed a clear interest in online data collection. Nearly all listed fast data collection and large sample sizes as benefits of online data collection, with most interested in the potential for lower costs and a more diverse population (Fig. 2). Of researchers who had reviewed a paper which included data collected online, $59 \%$ treated the data identically to data collected in the lab.

Respondents also felt that online data collected presented a set of unique challenges (Fig. 2). Some felt that the data collected online was unreliable, or the population unrepresentative. Many expressed that the experiment designs they were interested in did not work well online, or that web technology is too complex. Researchers based outside the US reported difficulty using services like Amazon Mechanical Turk.

Many respondents (56\%) listed the tool that they were currently most likely to use as Qualtrics, which is a service for conducting online surveys. However, a majority of participants (79\%) expressed interest in being able to run full experiments that included multiple trials which were timed precisely, measured reaction time, and other features typical of laboratory studies (Fig. 2). The vast majority (94\%) reported interest in new tools that simplified online data collection. A full list of the features researchers were looking for in a online data-collection system are shown in Table 1, along with a checklist showing which are currently handled by psiTurk. Importantly, psiTurk successfully meets many of the major challenges that researchers identified.

\footnotetext{
${ }^{1}$ The full dataset of participant responses is freely available at http:// gureckislab.org/data/online-data-survey.xlsx.
}

\section{psiTurk and Amazon Mechanical Turk}

psiTurk was primarily designed to facilitate online data collection using the Amazon Mechanical Turk (AMT) crowdsourcing platform, though it can also be used to run experiments in a traditional lab setting. Mason and Suri (2012) provide a general overview of AMT features of interest to academic researchers, one of which is that it provides a way for researchers to easily recruit and compensate individuals for online experiments. People who complete tasks (called Human Intelligence Tasks or HITs) are called workers, and people who post tasks are called requesters. Beyond providing a platform to connect requesters and workers, AMT places few restrictions on the type of work that can be requested. Workers are paid a fixed amount for each HIT they complete; this amount is determined by the requester and can be supplemented with bonus payments for good performance.

From a worker's perspective, the AMT website provides an up-to-date listing of HITs that are available, with a title, brief description, and payment amount. A worker can click on a HIT to see its full advertisement (an embedded webpage that is either hosted by Amazon or the requester) and decide to accept the HIT. Once the HIT has been accepted, the worker must complete it within a timeframe specified by the requester, or return the HIT so that it may be completed by a different worker. The HIT may be completed either on a requester's own hosted service (i.e., a webpage external to the AMT site), or on the AMT website itself through the use of customized templates. Upon completion, the worker submits the HIT, after which point the completed work must be approved by the requester before they are compensated. In addition, some HITs offer bonus payments 
Table 1 Features the surveyed researchers desire in a software system for online data collection

\begin{tabular}{|c|c|c|}
\hline Feature & $\%$ interested & psiTurk \\
\hline Ability to block participants from doing the same experiment twice & 90 & $\checkmark$ \\
\hline Ability to automatically pay people & 69 & $\checkmark$ \\
\hline Availability of example code that I can use to jump-start my own experiment & 64 & $\checkmark$ \\
\hline Ability to design experiment in the browser without programming (e.g., no Javascript or Flash needed)... & 64 & $\mathrm{X}$ \\
\hline No need to have a separate server/webserver installed and maintained & 61 & $\checkmark$ \\
\hline Ability to record information about browser (e.g. when people switch windows during task) & 60 & $\checkmark$ \\
\hline Ability to see if a online participant has done a similar study to yours in the past & 60 & $\mathrm{X}$ \\
\hline A graphical user interface for managing my online experiments (e.g., paying participants, viewing data) & 60 & $\mathrm{X}$ \\
\hline Open-source software I can read/edit myself if needed & 60 & $\checkmark$ \\
\hline Ability to automatically assign bonuses based on performance & 58 & $\checkmark$ \\
\hline Ability to run multi-day experiments where people come back for multiple sessions & 57 & $\bullet$ \\
\hline Ability to easily coordinate the running of multiple experiments at the same time & 55 & $\checkmark$ \\
\hline Ability to save data from experiment incrementally (in case browser or network crash can get partial data) & 53 & $\checkmark$ \\
\hline Automatically fill in conditions randomly and evenly & 52 & $\checkmark$ \\
\hline Ability to store data from my experiments in the cloud and have them automatically backed up & 50 & $\bullet$ \\
\hline Ability to obtain geographic and visualize geographic information about where participants are connecting from & 50 & $\mathrm{X}$ \\
\hline Ability to run group experiments (i.e., multiple people interacting) & 49 & $\bullet$ \\
\hline Tools to help document payments for reimbursement from University or Business (i.e., accounting issues) & 48 & $\mathrm{X}$ \\
\hline Other & 10 & \\
\hline
\end{tabular}

$\checkmark=$ provided by psiTurk, $X=$ not currently provided, $\bullet=$ possible with psiTurk, requires significant effort

that are determined by the requester based on a worker's performance.

From a requester's perspective, AMT provides a webbased interface for creating/managing HITs and approving work that has been submitted. In addition, it offers a sandbox version of the platform in which HITs can be tested without paying live participants. For basic tasks like surveys and open text fields, AMT offers a range of built-in templates that can be used to create experiments within the AMT website. However, the degree of customization is limited for these templates. In particular, they do not support advanced experimental logic that is common to many psychology experiments (e.g., adapting the presentation of stimuli based on performance, or counterbalancing across a number of experimental variables). In addition, they are limited to relatively simple forms of interaction and stimulus presentation.

For more complex tasks, a requester can provide a link to their own hosted web page or web application. This external HIT only interfaces with AMT at the beginning of the task (receiving basic information about the worker) and at the end of the task (sending confirmation that the task has been completed); the requester's web application is responsible for otherwise running the experiment and saving the data. Thus, external HITs enable a much wider range of experiments at the cost of additional technical requirements.

\section{What technical challenges is psiTurk designed to solve?}

The psiTurk platform is designed to handle many of the requirements associated with online experimentation-in particular, external HITs on AMT-so that researchers can run complex and sophisticated experiments more efficiently.

\section{Web server and database management}

To collect experimental data on the web, or specifically to run an external HIT on AMT, researchers minimally need a web server and database. The role of a web server is to respond to requests from a participant's Internet browser for the experiment content. The role of the database is to save the data provided by the participant. Although less common in laboratory experiments, databases are necessary for online studies because it is impossible to save data to a participant's computer using a web page or web application - data must be stored on the server, rather than the client. In addition, multiple participants often complete an experiment concurrently which can result in file corruption if two people try to write to the same file at the same time. Databases are specifically designed to mitigate this issue by managing concurrent reading and writing. 
Setting up a web server and database can be complex. Some universities and colleges provide these types of services, but the technical infrastructure at other institutions is lacking. psiTurk includes a custom webserver and database as a basic feature of the software tool which can be run from any computer including a standard office computer or laptop. This allows data collection from anywhere without the need for a dedicated server computer. In addition, psiTurk can be installed on free hosting services such as Amazon Web Services or RedHat's OpenShift. Data collected with psiTurk is stored at a location chosen by the experimenter and never visible to the psiturk.org cloud services or Amazon, protecting the privacy of participants and helping with IRB compliance.

An additional complication is that contemporary web browsers are increasingly security focused. In particular, certain browsers will not load content that is not properly signed with a SSL certificate and accessed with a https:// request. Such certificates are often difficult to obtain from universities and can be costly from online hosting companies. psiTurk solves this problem on behalf of researchers by using a cloud-based Secure Ad Server which presents securely signed content for users. One way to think of the Secure Ad Server is as a digital version of a bulletin board which is used to advertise particular studies within a university.

\section{Communicating with AMT}

When using AMT to recruit and compensate participants, experimenters typically use the AMT website. This often involves a lot of manual, error-prone clicking. To mitigate this, psiTurk has scripted many of the common tasks that researchers have to do on AMT's website, including paying all participants, assigning bonus payments, checking the current account balance, and monitoring the progress of an experiment. These tasks are performed via an interactive UNIX-based command line tool, which helps to prevent errors in payments and by saving researchers considerable time.

\section{Programming an experiment}

psiTurk provides a number of features which help researchers implement experiments. These include a JavaScript library (psiturk.js), basic HTML templates for commonly used components (e.g., consent forms and instruction screens), and a user-contributed Experiment Exchange where researchers can share the code for their psiTurk-compatible projects (http://psiturk.org/ee).

The psiturk.js JavaScript library provides basic functions for recording data and sending it back to the psiTurk server, preloading pages and images, as well as some basic functions for looping through instructions screens and providing (and recording) informed consent. However, the psiTurk framework is not designed as a generic programming interface for building experiments (e.g., it does not provide code for widgets such as sliders or buttons) and assumes researchers will use some basic JavaScript, HTML, and CSS to design their experiment. psiTurk's philosophy is that general purpose JavaScript libraries available online for creating dynamic content (e.g., jQuery or Twitter's Bootstrap library) provide the most powerful and well-documented set of features.

Rather than reimplementing the functionality of existing libraries, psiTurk aims to help share code and propagate "best practices" among the research community. These goals are supported by the online Experiment Exchange, which catalogues existing psiTurk experiments. The source code for projects in the exchange can be downloaded and modified for a new use, replicated directly, or can simply provide example of code implementing a particular feature. In addition to the exchange, psiTurk also includes a complete working example experiment that can be used as a template for new projects.

Examples of experiments already shared in the Experiment Exchange include mental rotation experiments, feature rating tasks, interactive drawing tasks, auditory lexical decision and identification tasks, decision making experiments, and other common designs in cognitive psychology. Any interface that can be programmed in a browser can in theory be adapted for use with psiTurk which means anything from surveys to rich video-game type interfaces are possible.

\section{Recruiting, filtering, and instructing participants}

Finally, psiTurk has a number of features for controlling the recruitment of participants which can contribute to higher quality control. For example, psiTurk offers basic capabilities for assigning workers to different experimental conditions and counterbalancing different aspects of the experiment. In addition, it allows experimenters to filter workers in particular ways. For example, through psiTurk, is it possible to exclude participation by workers using certain devices (such as phones or tablets) or browsers which are known to have compatibility issues with the experimenter's design (for example Internet Explorer). psiTurk also allows researchers to prevent a worker from participating in the same experiment more than once (which might otherwise be tempting when large financial rewards are offered). Future versions of psiTurk may also allow researchers to block AMT workers who have completed certain psiTurk experiments in the past, such as any experiment with a similar task (see Chandler 2014, for a discussion about non-naivety amongst AMT workers). 


\section{Key components of the psiTurk platform}

In the last section, we described the technical challenges that psiTurk was designed to address. Now, we describe how these challenges are actually met by giving a more detailed overview psiTurk's different components.

The overall structure of psiTurk is shown in Fig. 3 and can be conceptualized as involving three components. First, there are a collection of software tools which run locally on a user's computer or server. These local components include a command line tool and a web application server (which provides both a customizable webserver and database solution). Second, psiTurk relies currently on Amazon Mechanical Turk to handle the recruitment and compensation of participants. Third, the psiturk.org cloud based component includes a hosted Secure Ad Server and the Experiment Exchange. In the following sections we highlight the important functions of each component.

\section{Local computer or server}

\section{Command line tool}

When using psiTurk, the researcher runs an interactive UNIX-based command line interface (CLI) on a local computer, such as a laptop or lab computer. This tool is used to issue commands, and to interact with the Amazon Mechanical Turk system and with the psiTurk cloud. Example uses of the command line tool include checking the user's Amazon Mechanical Turk account balance, paying participants, and creating new HITs. In addition, the command line has the capability to give automated bonus payments to participants based on a function designed by the experimenter. This feature is particularly helpful since the AMT website requires bonuses to be entered by hand for each participant individually.

There are several advantages to designing psiTurk as a simple CLI beyond its efficiency of use. This design makes the user interface code clear and easy to read and write, allowing newcomers to quickly understand and contribute to the open-source project. Integrating a new feature into the interface is as simple as describing the syntax and functioning of a new command; in contrast to a graphical user interfac (GUI), extending the CLI does not require designing time-consuming GUI elements. The CLI also ensures that psiTurk is easy to interact with not just on a laptop or desktop, but also on a remote server or in the cloud, where users may only have terminal access.

\section{Web application server (webserver + database)}

The command line tool is also used to interact with the web application server. The web application server handles the logic determining which pages to show to a participant at different stages of the experiment. psiTurk comes with a default web application which implements many important features commonly needed by experiments (e.g., checking that a user hasn't already done the experiment, recording and saving data to a centralized database). In addition, the web application itself can be heavily customized to support functions that are difficult to code in JavaScript, or easily exploitable by savvy participants. Such customizations might be as simple as changing the way that bonuses are assigned to participants, but they can also allow for much more complex experimental designs. For example, psiTurk allowed us to create an experiment where a cognitive model is fit to participant's data in real time and used to determine the sequence of future trials in the experiment.

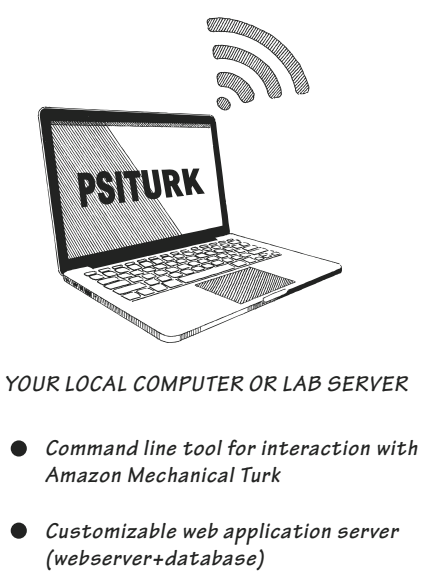
(webserver+database)

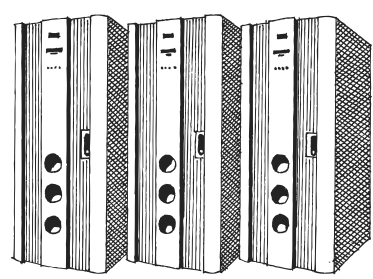

amazonmechanical turk

- Handles recruitment of participants

- Transfers money between Requester and Worker Accounts

- Provides a listing of available HITs

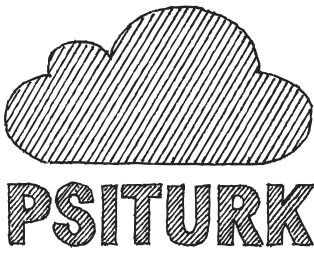

THE PSITURK.ORG CLOUD SERVICES

- Secure Ad Server (provides a free hosting service for "experiment ads" which appear in the AMT listing of available HITs)

- Experiment Exchange (an open-source repository of experiments which can be easily downloaded)

Fig. 3 The three core components of the psiTurk system 


\section{Amazon Mechanical Turk and Amazon Web Services}

Amazon Mechanical Turk's servers handle the recruitment of participants via its crowdsourced labor force. It also handles the transfers of money between Requesters and Workers. Finally, it provides a listing of available HITs which workers can browse through. Amazon Mechanical Turk is one part of a larger collection of cloudbased services provided by Amazon via the Amazon Web Services. For example, by using the psiTurk command line tool, users can create a database server running on Amazon's cloud with a few short commands. This database can then be used instead of a local database solution.

\section{psiturk.org cloud services}

In addition to the command-line tool and the web application server, psiTurk provides a few web services that are continuously running on a separate web server. Most important of these is the psiTurk Secure Ad Server. As mentioned earlier, when an experimenter creates a new HIT and posts it to AMT, they need to display an $a d$ explaining how long the task will take and how much it will pay. However, ads will not correctly display in workers' browsers unless they are encrypted using SSL. To resolve this issue, the Secure Ad Servier allows users of the psiTurk system to host ads on the psiturk.org website which are encrypted.

Besides the convenience of the Secure Ad Server in terms of providing SSL signed URLs, the Secure Ad Server tracks statistics about the number of workers who viewed the ad but decided to not accept it, the number who accepted it, and the number who actually completed the task. These statistics are made available on the psiturk.org dashboard and can be an additional source of information for experimenters.

Finally, in addition to the Secure Ad Server, the psiTurk Experiment Exchange provides a open-source repository of psiTurk-compatible experiments which can be easily downloaded. The coordination of these downloads is handled by the psiturk.org cloud.

\section{Requirements and initial setup}

This section describes the requirements and installation steps that are required to successfully setup psiTurk and run HITs on Mechanical Turk. Note that details of these steps may change over time and will be updated at http://psiturk.readthedocs.org.

\section{System requirements}

psiTurk is designed to run on UNIX systems (including Mac OS X, BSD, and Linux) that satisfy the following requirements:

1. A working installation of Python 2.7 (Python 3 is not currently supported)

2. The pip package manager

3. A command line tool (like Terminal in Mac OS X)

4. A web browser (ideally one that provides web developer tools, like Firefox, Safari, or Chrome)

psiTurk relies on a variety of open-source packages developed for Python. Most centrally this includes Flask (http:// flask.pocoo.org/) which is a micro framework for developing web applications in Python, gunicorn (http://gunicorn. org/) a Python-based HTTP server, and boto (https://boto. readthedocs.org/en/latest/) a Python interface to Amazon Web Services. There are a variety of other packages which provide supporting functions which are automatically installed using the pip package manager when psiTurk is installed. However in many ways psiTurk is a package which glues together the functionality of Flask, gunicorn, and boto. These packages were selected due to their relatively mature development status, their open-source licensing, and their ease of use. To satisfy the Python requirements, we recommend using an existing distribution like Enthought or Anaconda. These are free Python distributions that ship with a range of useful packages, including the pip package manager.

\section{AWS credentials}

An Amazon Web Services (AWS) account is required to use AMT as a requester. A new account can be created at http:// aws.amazon.com/mturk/. To set up an account, note that requesters must provide a U.S. billing address, so this may bar researchers outside the U.S. from creating an account.

After setting up an account, psiTurk users must retrieve their AWS credentials, which include an access_key_id and a secret_access_key. These can be retrieved and (re-)generated at https://console.aws.amazon.com. These credentials will be entered in the .psiturkconfig file, as explained below.

\section{psiTurk credentials}

Additionally, a psiTurk account must be created at https:// psiturk.org/register, which allows users to post ads on psiTurk's Secure Ad Server. A new user must then obtain a set of psiTurk credentials which can be found in the 
"API Keys" menu item in the drop-down at the top right hand of the page. These credentials will also be placed in .psiturkconfig (see below).

\section{Installation}

psiTurk is installed using the Python package manager pip. In a terminal window, type ${ }^{2}$ (excluding the dollar sign):

\$ pip install psiturk

Alternatively, psiTurk can be installed directly from GitHub, which holds the latest stable release in the master branch, and the latest development version in the dev branch. To install from master, for example, type:

$\$$ pip install

git+git://github.com/NYUCCL/psiTurk.git@master

The psiTurk installation automatically creates a global config file, .psiturkconfig, in a user's home directory. This file must then be edited to include the AWS and psiTurk credentials of the specific user.

\section{Optional: Adding a SQL database}

By default, psiTurk includes a basic database solution called SQLite. This solution will work for HITs that only require light traffic, but it does not handle multiple concurrent queries well: there is a danger of data loss when a SQLite database is accessed at the same time by different users. It is therefore recommended to set up a more robust database solution, like a MySQL database, especially if a researcher wants to run many participants simultaneously. Fortunately, Amazon's Web Services (of which Mechanical Turk is just one component) provides a relatively simple and inexpensive way to set up a SQL server. Please refer to the documentation at http://psiturk.readthedocs.org/ ("Configuring Databases" section) for complete instructions.

\section{Getting started with psiTurk}

The following section provides a basic overview of how to use psiTurk, explaining the various steps required of new users of the system along with some details about how the various components described above interact.

\section{Configuration and project structure}

A new psiTurk project can be initialized in the current directory using (without the dollar sign):

\$ psiturk-setup-example ${ }^{2}$ Depending on the user's permissions, this command may have to be
prefaced with sudo.
This creates a new directory containing an example experiment, which can be used as a template for more sophisticated designs. The project directory typically includes the following files (though some are only created after the experiment is run for the first time):

- config.txt: a text file containing settings for the current experiment, including:

- Metadata about the experiment that is displayed to workers on the AMT website (title, description, etc.)

- Restrictions on which workers can accept the HIT, including geographic location (e.g., limiting to US workers only), AMT-specific qualifications (e.g., a worker's proportion of past work that has been approved), and web browsers that are permitted.

- Database information (SQLite, by default)

- Server parameters (URL, port number)

- Task parameters (e.g., number of conditions)

- custom.py: a Python file containing user-provided custom functionality for the psiTurk web server

- participants.db: the SQLite database (if SQLite is being used)

- server. log: a log of any messages from the experiment server (not from the actual experiment code)

- static/ a directory containing experiment files, including JavaScript, CSS, and images.

- templates / a directory containing HTML files associated with the experiment

Setting up a new experiment thus entails 1) editing the settings in config.txt, and 2) modifying the contents of the static and templates directories to reflect your experimental design (note that programming the experiment requires at least some basic web programming skills, including HTML, CSS, and JavaScript).

The remaining files are only necessary in order to debug server errors (server.log), add new serverside functionality (custom.py), or to access saved data (participants.db).

\section{Command line interface: Managing HITs and serving the experiment}

As described in section "Command line tool", psiTurk runs as a command line interface (CLI) within a standard terminal window and can be used to perform a wide variety of tasks-from creating HITs and paying workers, to launching Amazon Web Services database instances, to opening an experiment in a browser for debugging - that would otherwise be spread across a number of websites and programs. In most cases, a user of the psiTurk CLI will never have to 
$\log$ into the MTurk website except to add money to their MTurk requester account and to first create the account (and to accept the terms of service).

Entering psiturk at the command line in any directory containing a psiTurk project launches the psiTurk CLI, which features a colorized prompt that provides important information at a glance, including whether the server is running, the current mode (live or sandbox), and the number of HITs currently running on AMT in the same mode:

[psiTurk server:off mode:sdbx \#HITs:0]\$

In some examples below, this prompt is truncated as [psiTurk] \$ to indicate commands that occur within the psiTurk CLI, while commands that are entered at the user's standard command line are preceded simply by \$.

\section{Example CLI usage}

Commands are organized into groups based on their function, following a general command subcommand arguments format. For example, one can create a HIT by typing:

\section{[psiTurk] \$ hit create}

which launches an interactive prompt for number of assignments, payment per HIT, and HIT duration. The sandbox mode is active by default, which means that calling hit create will submit a HIT to the AMT sandbox website.

After creating one or more HITs, one can list any active HITs with:

[psiTurk]\$ hit list --active

The experiment server is launched using:

[psiTurk]\$ server on

which opens the port specified in the project configuration file, config.txt. The experiment server will then respond to incoming requests, assuming that the port is publicly accessible. This requires a static IP to prevent the experiment's URL from changing. Users without a static IP address can use a dynamic DNS service to forward requests to their dynamic IP. If the system running psiTurk is behind a router, the router must be configured to forward requests on the same port.

During the development of an experiment, the current code can be tested using:

\section{[psiTurk]\$ debug}

which will open a new browser window in which the current experiment can be tested. The experimenter can then test the experiment from the perspective of a worker before making it publicly available. Once the experiment is ready for real workers, the mode command can be used to switch to live mode, after which newly created HITs will be submitted to the main AMT site.

Help on any of the commands is easily accessible through the help command. Using help on its own will print all available commands, and using help command will print out detailed help on the given command.

\section{The Secure Ad server}

As described briefly in section "Web server and database management", the psiturk.org cloud service provides a Secure Ad Server. The term ad in this context refers to a small HTML page which describes the details and requirements of a given experiment. The ad is the first interaction that an experimenter has with a potential subject and is thus the gateway to subject recruitment. Ads are in many ways analogous to hanging a poster or flyer around a university building in order to recruit participants. It's easy to overlook the importance of a good ad, and making that ad visible to as many participants as possible.

An overview of the flow of running an experiment using psiTurk and the Secure Ad Server is illustrated in Fig. 4. When a researcher using psiTurk runs the hit create command, the current contents of the local templates/ad.html file are uploaded to the psiturk.org cloud (see the right panel of Fig. 4). This will create a unique custom URL for the experiment (an example might be https://ad.psiturk.org/view/90812390123091823) which allows anyone on the internet to view the contents of the uploaded ad.html file. Critically, the ad URL is encrypted and securely signed with an appropriate SSL certificate ensuring that all web browsers will successfully display the webpage. ${ }^{3}$ A different URL is created each time hit create is run and there is no limit to the number of ads that can be created. A private listing of all ads created by a particular user is available on the psiturk.org dashboard (https://psiturk.org/dashboard).

The custom ad URL is automatically passed on to AMT by the hit create command as the location of the selected HIT. As is visible in Fig. 4, when an AMT worker views a HIT on the AMT website, it loads the ad from the psiturk.org cloud server (left panel of Fig. 4). If the worker accepts the hit, a link is provided to the worker which pops open a new browser window. This URL points directly at the location of the requester's local computer or server (right panel). After this hand-off occurs, all data is transmitted directly between the worker and the requester: the psiturk.org cloud server does not ever process or see the user's experimental data. This is important for many IRBs, particularly if an experiment involves sensitive materials.

\footnotetext{
${ }^{3}$ SSL certification has recently become a requirement for hosting content within an iframe on mturk. com.
} 


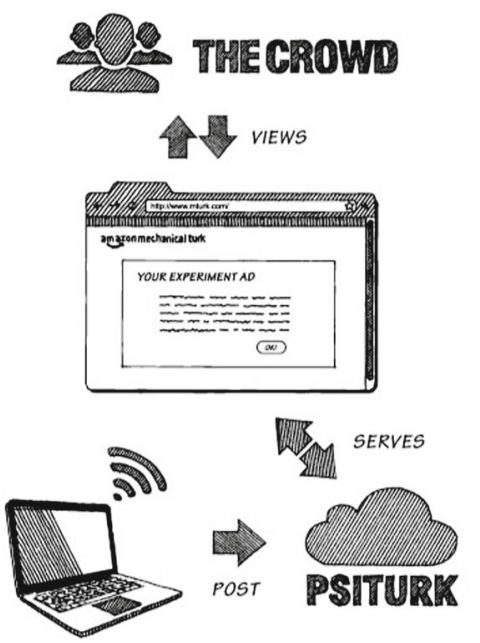

Fig. 4 How the Secure Ad Server works

One possible complication with this approach is that if the computer hosting the experiment goes down any participants currently taking the experiment may encounter connection problems. Since psiTurk encourages a distributed model where the webserver runs on a local machine this can happen accidentally (e.g., if a laptop computer running psiTurk loses a wifi connection). Although this problem affects all approaches to web-based experimentation, the lack of a dedicated server model can exacerbate this concern. However, it should be possible in most cases to restart the server and allow participants to continue (assuming the server interruption is temporary). If the local psiTurk server is offline or unreachable, new participants will not be able to begin the experiment because he ad server communicates with the psiTurk researchers local machine during the display of the ad to verify it is still online.

\section{JavaScript library psiturk.js}

The JavaScript library psiturk.js enables interaction with the server from the client-side (JavaScript) experiment code. The goal of the library is to handle the most common functionality of psiTurk-based web experiments, without imposing any additional requirements on the structure or design of the experiments themselves. Researchers can use any third-party JavaScript libraries to design their experiment, while relying on psiturk.js for common functions, including saving data and notifying the server of changes in a participant's status.

Listing 1 shows a condensed version of the JavaScript code from the Stroop example experiment. This is used in the following sections to illustrate how experiment code uses functions from psiturk.js in order to interact with the psiTurk server. For the full, functional version, see the file static/js/task.js in the example experiment directory.

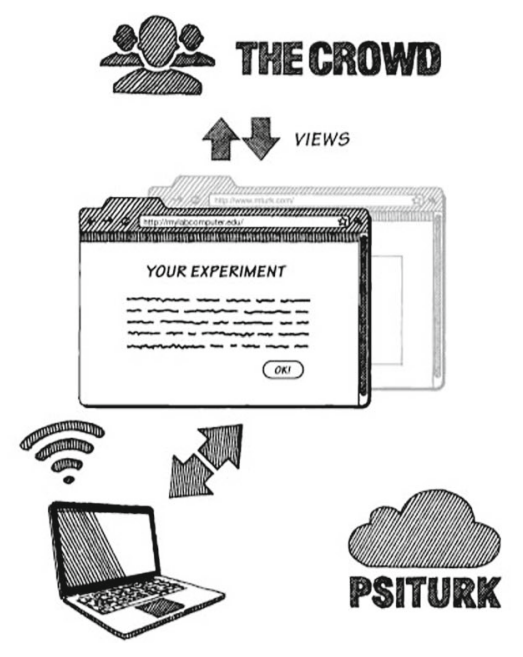

Initialization

When the experiment files are requested from the psiTurk server, the following global variables are also set (see templates/exp.html):

- uniqueId: a unique identifier for the participant

- adServerLoc: the experiment-specific URL on the Secure Ad Server

- mode: the current mode (debug, sandbox, or live)

- condition: experimental condition

- counterbalance: counterbalancing code, used to vary design elements that are not related to main manipulation

A psiTurk object must be initialized based on these global variables (line 2). These variables can also be used to change the experimental logic. For example, a common requirement is to change the stimulus set or design based on the condition or counterbalance codes that have been assigned by the psiTurk server (lines 4-5).

Following instantiation, the psiTurk object can be used to preload a list of HTML pages that reside in the templates directory of the experiment (lines 8-9). This allows a new page to be displayed using a single command (line 34), which replaces the body of the window with the content in the specified HTML file. The psiTurk object also has functions for preloading images (in order to avoid server requests in the middle of the experiment when an image is displayed) and a basic structure for presenting a series of HTML pages (e.g., for an instructional phase).

\section{Tracking a participant's progress in an experiment}

psiTurk records changes in a participant's status as they move through an experiment. Some of these status changes 
are automatic, e.g., when a participant is assigned to a condition or if they quit an experiment early. Two additional status changes are initiated by the client-side experiment code using psiturk.js functions. First, since experiments typically begin with an instructional phase, calling the function psiTurk. finishInstructions () upon completion of this phase will save the participant's status as having begun the main experiment. If the participant attempts to quit the experiment after that point, they will receive a warning that they will not be able to restart the experiment and may forgo payment. Second, successful completion of the experiment is signaled by calling psiTurk.completeHIT(), which closes the experiment and redirects the participant to the AMT page to submit their HIT.

\section{Saving experimental data}

The experimenter can save data in two formats. psiTurk.recordTrialData takes any JSON object as input and appends it to a list of trial data. This data structure is meant for sequential data that may be collected over the course of multiple trials or blocks, where each line corresponds to a new measurement. However, the format of each entry is defined by the experimenter and is saved in the database as a single JSON object (see line 20). Each new entry is automatically time-stamped at the time that psiTurk.recordTrialData is called.

In contrast, psi Turk . recordUnstructuredData is used to record (key, value) pairs, where the key is uniquely defined within the experiment (see line 43). This format is useful for survey questions, randomly generated experiment parameters, or other one-time measurements, e.g., (Age: 24).

Importantly, both functions above simply record the data in the appropriate format on the client-side (i.e., within the psiTurk JavaScript object). When the experimenter wishes to save the data to the server they call psiTurk. saveData (), at which point both sets of data are sent to the server to be recorded in the database. psiTurk.saveData () can also be called with functions that will be executed upon either success or failure of the server request. For example, if the final save request is successful, the experiment can be terminated with psiTurk. completeHIT() passed as a callback (see lines 56-63).

\section{Automatic recording of browser interactions}

One general limitation of web experiments is greater uncertainty about a participant's testing environment and engagement with the experiment. Unlike lab computers where most undesired behaviors can be prevented, a web participant is always able to close a web browser, switch to different applications, or change other aspects of their experience. However, standard methods exist for recording a user's interaction with a web browser, and these data can be useful for 1) tracking how an experiment was actually displayed, and 2) the level of a participant's engagement.

For example, although it is possible to control the initial size of a browser window, a web participant can change the dimensions of the window, potentially obscuring or altering the displayed elements. psiturk.js automatically records these changes in the size of the window. Similarly, the participant can choose to switch focus away from the experiment window (e.g., to another browser window or a different application). psiturk.js automatically records every time that the experiment window loses and gains the participant's focus. This event data is saved to the database whenever psiTurk.saveData is invoked, without any additional action on the part of the experimenter.

\section{Experiment Exchange}

A significant advantage of web-based experiments is the potential for low-friction replication and extension. The Experiment Exchange (https://psiturk.org/ee) facilitates the sharing of experiments that have been built to run using psiTurk, acting as an "app store" for psiTurk-compatible designs. Once an experiment has been completed, a researcher can submit the following information to register their experiment on the exchange:

1. A GitHub repository containing the project code

2. Metadata about the experiment, including a title, description, and keywords

3. The DOI for the paper describing the results of the experiment (if any)

4. The version of psiTurk that was used to run the experiment

Metadata associated with an experiment allows other researchers to discover it through the psiTurk website. A publicly available experiment can then be downloaded using the following command in the terminal:

\$ psiturk-install <experiment-specific-id>

with the experiment-specific identifier found on the exchange page. If psiTurk is already configured on the user's system, the downloaded experiment will run without further changes, and can then act as a starting point for direct replication or extensions. Modified versions can then be re-run using the same population via AMT, thereby minimizing the potential for experimenter-specific biases. 
Other initiatives (e.g., the Open Science Framework, https://osf.io) also aim to improve the transparency, reproducibility, and efficiency of research through centralized services, but are less focused on the specific technology stack used to run online experiments. In contrast, the psiTurk Experiment Exchange links together experiments that can be run within a common framework. As a result, existing experiments can serve as examples to help new researchers learn to code for the web; they shorten the development time necessary (especially for popular experimental paradigms); and the exchange facilitates communication between researchers with similar interests.

\section{Adoption by the community}

At the time of publication of this paper, there are 444 registered users accounts on https://psiturk.org. So far 24,203 distinct Amazon workers have completed psiTurk tasks and 9,107 attempts to complete the same experiment more than once have been blocked. The psiTurk command line tool itself has been launched 42,648 times. In addition, the source code for ten psiTurk-compatible experiments have been published in the Experiment Exchange including some by researchers who are not authors of this article.

\section{Related projects}

There are a variety of software projects related to psiTurk. WEXTOR is a Javascript-based experiment generating system which helps researchers with little programming experience develop experiments (Reips \& Neuhaus, 2002). psiTurk has similar aims but allows for greater customization and more direct interfacing with Amazon Mechanical Turk. TurkGate (http://gideongoldin.github.io/ TurkGate/) is a project to help researchers group surveys together to prevent repeat participation by Mechanical Turk Workers. However it doesn't directly deal with paying participants, providing a webserver, or storing participant data. Turkserver-Meteor (http://turkserver.meteor.com/) is a framework for building real-time web apps that interface with Mechanical Turk and provides many similar features to psiTurk but is based on the Meteor development platform.

\section{Limitations and future directions}

psiTurk solves a number of the challenges facing researchers interested in online data collection. However, at the time of the publication of this article, there remain a number of limitations that remain to be solved.
One possible limitation is that psiTurk is currently designed to work primarily with Amazon Mechanical Turk. A common worry with AMT is the unrepresentativeness of its subject pool (in terms of demographics and geography), as well as the fact that certain experimental protocols are now too well-known to workers to guarantee naive participants (Chandler et al., 2014). Indeed, some of the concerns raised by survey respondents in section "The challenges of web-based experimental behavioral research" are inherent to online data collection or the AMT platform and therefore psiTurk does not address them. It is worth pointing out, however, that the common concern about data reliability may be somewhat exaggerated based on recent studies that have successfully replicated a wide range of classic cognitive psychology findings using AMT data (Crump et al., 2013). Interestingly, this study also found that increasing worker payment had no effect on reliability, suggesting that even at low payment levels data quality was high.

To address some of these concerns, some experimenters may look for alternatives to AMT. Many psiTurk features (i.e. the web server, data base, and psiturk.js) are general enough to facilitate running experiments on other platforms, too, but it would require some customization of certain AMT-specific functionality. Researchers who decide to use psiTurk on another platform could contribute such changes to the GitHub repository (https://github.com/NYUCCL/ psiTurk) to make them available to the community.

There also clearly exist experimental protocols that simply are not amenable to online experimentation. Experiments that require very fine-grained temporal control over stimulus presentation, for example, may be unsuited because browsers will not be able to reliably display content fast enough if the presentation time becomes too short (Crump et al., 2013). Similarly, any experiment that requires control over a participant's screen size, resolution, or distance from the screen will be problematic due to the nature of the web browsing environment. One advantage of psiTurk is that it automatically collects data on worker's interaction with the experiment window, that is, if and when the window was resized and if and when the user switched tabs or windows. These kind of data can be used to evaluate a worker's level of engagement while completing the task. Nevertheless, the general lack of control over, for example, who drops out of an experiment, the environment of the participant, and the degree of motivation that participants bring to a task may present serious challenges and render online experiments unsuitable for some. To help researchers decide whether online experimentation is an appropriate method for them, we refer the reader to previous work that focuses in more detail on the various and in some cases unavoidable differences between lab and online experimentation (see e.g., Reips, 2002; Birnbaum, 2004). 
// Initalize psiturk object with parameters passed from server (see templates/exp.html) var psiTurk = new PsiTurk (uniqueId, adServerLoc, mode);

var mycondition = condition; // these two variables are passed by the psiturk server process var mycounterbalance = counterbalance; // they tell you which condition you have been assigned to

// All HTML snippets to be loaded from templates directory

var pages $=[$ "instructions/instruct-1.html", ...];

psiTurk.preloadPages (pages);

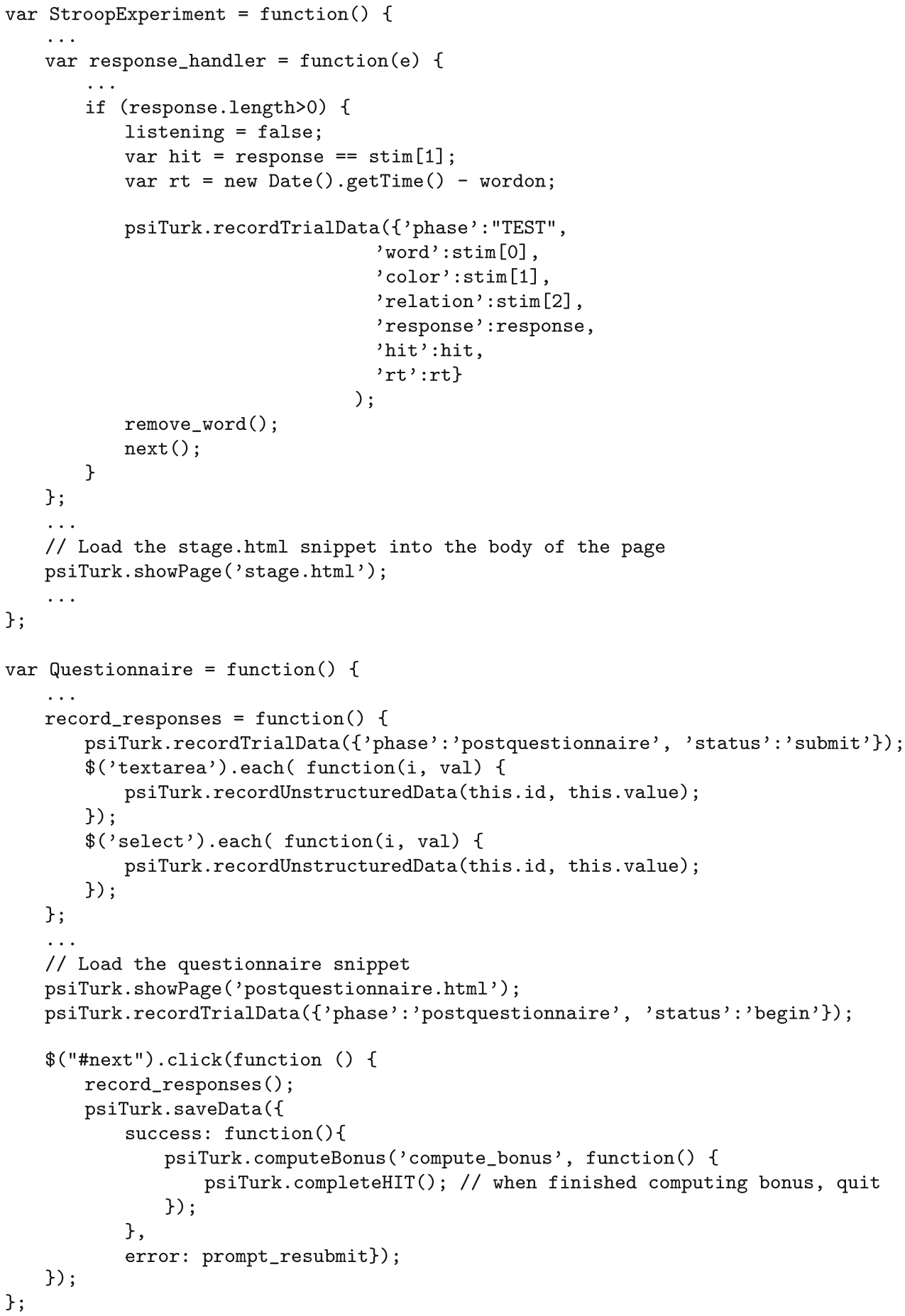

Listing 1 Condensed version of JavaScript code for Stroop experiment 
Another limitation is that psiTurk only runs on UNIX systems, like Linux and Mac OS, and can thus not be used on Windows computers. To meet this challenge, one option for Windows users is to use a cloud-based computing service to run psiTurk and host experiments. There exist multiple free or low-cost options, such as Amazon Web Services (https://aws.amazon.com). The installation steps and setup may diverge slightly from the standard procedure but the psiTurk documentation already contains additional installation instructions for OpenShift. In the future, we hope to extend support and documentation to a wider range of cloud computing options.

Another future direction is to extend the counterbalancing capabilities of psiTurk. Currently, the built-in counterbalancing algorithm simply aims to assign participants equally to different cells. However, this equal assignment method can be problematic if, for instance, one group has a higher drop-out rate than the others (a problem rarely encountered in the lab). In that case the high drop-out group will need to receive more participants to equalize the difference and thus assignment probabilities will no longer be equal across groups. We are currently working on alternative counterbalancing algorithms that keep assignment probabilities equal (which may then lead to unequal number of participants) to avoid this problem.

One exciting prospect of running online experiments is to let multiple participants interact to study coordination or group behavior. Currently psiTurk does not offer its own tool to facilitate such multi-person experiments, but we would like to add support for it in the future, using web-sockets or other protocols that enable communication between users.

\section{Conclusions}

As online data collection becomes more widespread, new software tools will need to be created. While in some cases these tools may come from for-profit enterprises, the project described in this paper advances an open-source, community-driven approach to software design. A core philosophy is that better scientific software developed can be developed with more diverse input from many programmers (Raymond, 1999). The advantages are that the quality of the code can be improved by having many people read and help catch bugs. In addition, "best practices" can be more readily propagated through the research community through code share such as in the psiTurk Experiment Exchange.

\section{References}

Birnbaum, M. H. (2004). Human research and data collection via the internet. Annual Review of Psychology, 55, 803-832.

Chandler, J., Mueller, P., \& Paolacci, G. (2014). Nonnaïveté among amazon mechanical turk workers: Consequences and solutions for behavioral researchers. Behavior Research Methods, 46(1), 112130.

Chen, R., \& Sakamoto, Y. (2013). Perspective matters: Sharing of crisis information in social media. In 46th Hawaii 46th Hawaii International conference on systems sciences(pp. 2033-2041). IEEE.

Crump, M. J., McDonnell, J.V., \& Gureckis, T.M. (2013). Evaluating amazon's mechanical turk as a tool for experimental behavioral research. PloS One, 8(3), e57, 410.

Kramer, A., Guillory, J., \& Hancock, J. (2014). Experimental evidence of massive-scale emotional contagion through social networks. Proceedings of the National Academy of Sciences of the United States of America, 111(24), 8788-8790.

Mason, W., \& Suri, S. (2012). A guide to behavioral experiments on mechanical turk. Behavior Research Methods, 44(1), 1-23.

Open Science Collaboration (2012). An open, large-scale collaborative effort to estimate the reproducibility of psychological science. Perspectives on Psychological Science, 7, 657-660.

Raymond, E. (1999). The Cathedral and the Bazaar.: O'Reilly.

Reips, U.D. (2000). The web experiment method: Advantages, disadvantages, and solutions. Psychological Experiments on the Internet, 89-117.

Reips, U.D. (2002). Standards for internet-based experimenting. Experimental Psychology, 49(4), 243-256.

Reips, U.D., \& Neuhaus, C. (2002). Wextor: A web-based tool for generating and visualizing experimental designs and procedures. Behavior Research Methods Instruments, \& Computers, 34(2), 234-240.

Wu, S., Hofman, J., Mason, W., \& Watts, D. (2011). Who says what to whom on twitter. In International conference on the world wide web $(W W W)$. Hyderabad, India. 